\title{
All-optical switch based on nonlinear photonic crystals ring resonator
}

\author{
Yonghua Yuan ${ }^{1 a}$, Zhengbiao Ouyang $^{1 \mathrm{~b}}$ \\ ${ }^{1} \mathrm{THz}$ Technical Research Center, Shenzhen Key Laboratory of Micro-nano Photonic Information \\ Technology, College of Electronic Science and Technology, Shenzhen University, Shenzhen \\ 518060, China \\ a617986516@qq.com, ${ }^{\mathrm{b} C}$ Corresponding author: zbouyang@szu.edu.cn
}

Keywords: switch; ring resonator; grapheme; photonic crystal

Abstract. An all-optical switch based on nonlinear ring resonator in a photonic crystal is proposed in this paper. The size of the structure proposed is $18 \mathrm{a} \times 18 \mathrm{a}$, where $\mathrm{a}=0.609 \mu \mathrm{m}$ is the lattice constant of the device. Graphene is used as the nonlinear material and wave absorbing material. The optical switch has been investigated by finite difference-time domain (FDTD). In order to reduce the scattering and improve the coupling efficiency, four scattering rods and six coupling rods are set as composite layers with graphene material inside the ring resonator, respectively. The all-optical switch shows excellent performance for having short switching time and desirable contrast ratio, which are $10.43 \mathrm{ps}$ and $36.8 \mathrm{~dB}$, respectively.

\section{Introduction}

Optical switches have tremendous potential applications in optical computing, optical communication and signal processing. Many all-optical logic devices, such as AND, NOR, XOR logic gates and digital elements, can be built by using optical switches.

In this paper, a new kind of all-optical switch is realized based on a nonlinear ring resonator in a square-lattice photonic crystal. The photonic crystal, which has been designed with appropriate parameters obtained through analyzing its band structure by finite-difference time-domain (FDTD) method, is used to realize the ring resonator by removing two rows and one circle of dielectric rods. The ring resonator has been widely studied before [1-2] because of its special characteristics. Graphene, whose characteristics had been widely studied [3-7] since it was found by two physicists in 2004, is used as the nonlinear material and wave absorbing material in our optical switch. In the process of designing device, wave absorbing material is widely used to stop the reflection and leakage of electromagnetic wave, because reflection and leakage can cause interference between optical circuit components and other working units. In order to reduce the scattering and improve the coupling efficiency, four scattering rods and six coupling rods are set as composite layers with graphene material inside the ring resonator, respectively. We assume that the controlling light is normally applied from top surface. For different controlling light intensity, its nonlinearity is different, so that the photonic crystal ring resonator responds differently, resulting ON and OFF states of the switch.

\section{The structure and its analyses}

The whole device is based on a 2-port ring resonator, as presented in Fig.1. Signal inputs from port A and outputs from port B for optical switch. 


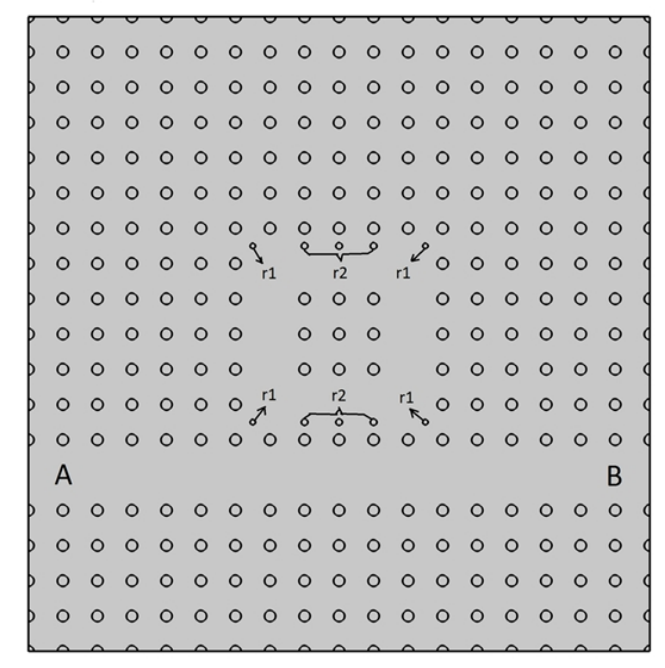

Fig.1. Structure of the optical switch

In order to reduce the scattering, a scattering dielectric rod is placed at each corner of the ring resonator, shown in Fig. 1 as r1. The six coupled dielectric rods denoted as r2, illustrated in Fig.1, are designed to improve the coupling efficiency when the switch is in ON state.

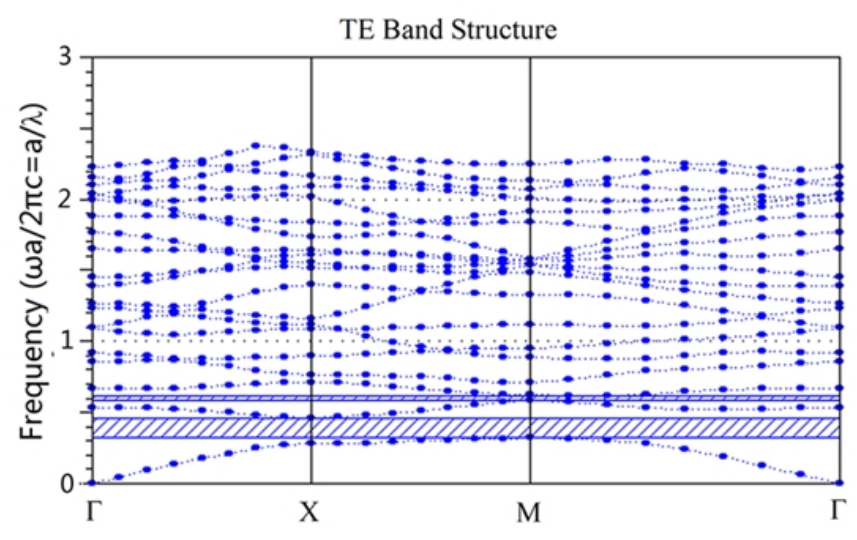

Fig.2. TE band structure of square-lattice photonic crystals

The TE band structure of square-lattice photonic crystals has been investigated, as shown in Fig.2, and the resonant modes and guided modes has been fully studied by analyzing the TE band structure before. The max normalized photonic band gap of our structure ranges from 0.324 to 0.464. We only consider the transverse electrical wave here. Firstly, we want to set the operating wave length of our optical switch to be $1.55 \mathrm{um}$.

\section{Simulation results}

The performance of our all-optical switch is controlled by assuming that a light normally applied to the device surface. In Figs. 3-5, the influence of controlling intensity, electric field distribution and switching performance are shown, respectively. 


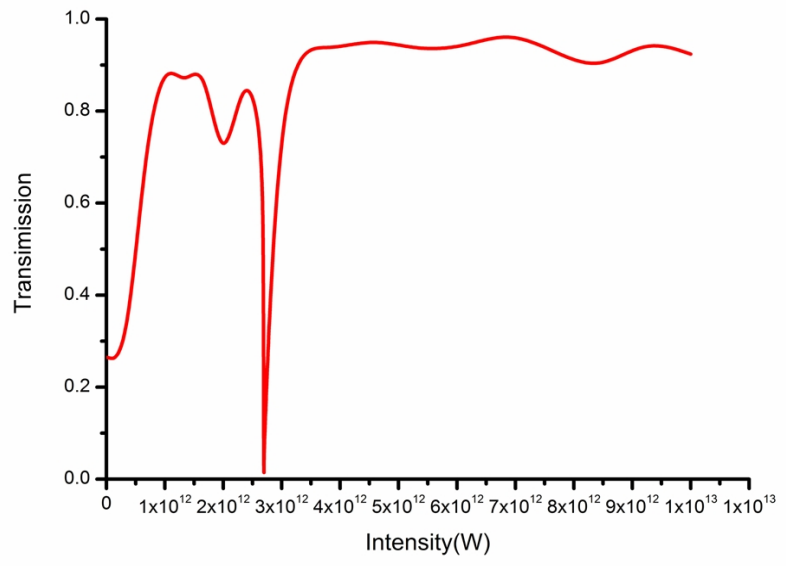

Fig.3. Influence of controlling intensity

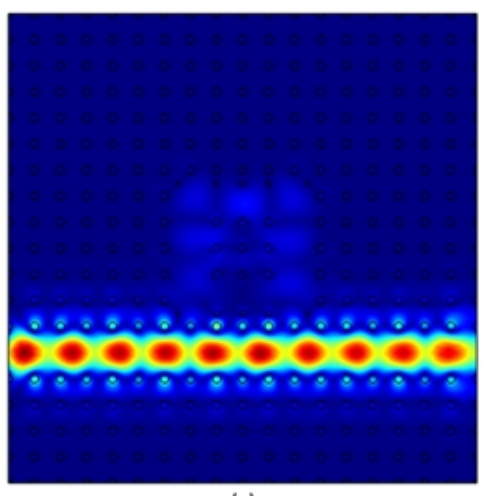

(a)

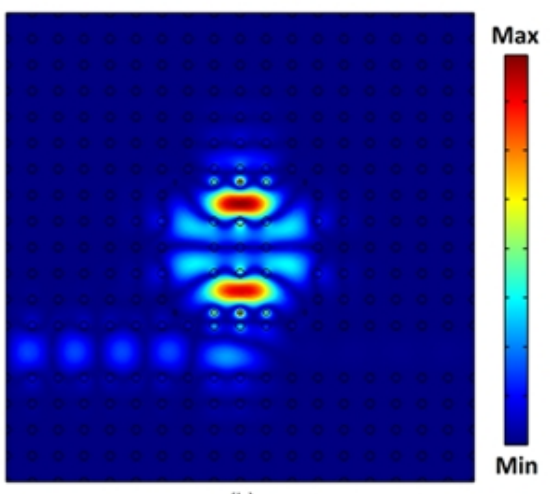

(b)

Fig.4. Electric field distributions in ON state (a) and OFF state (b)

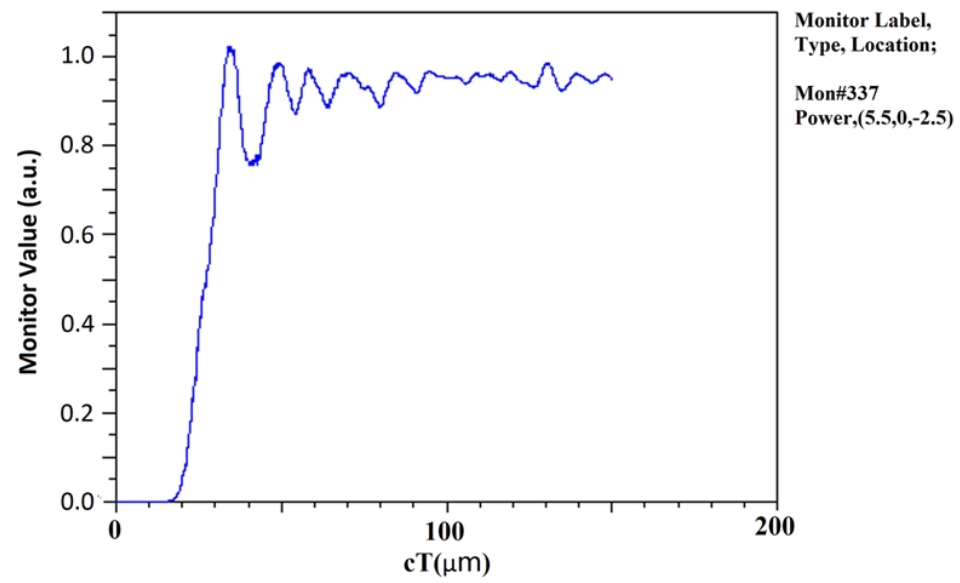

Fig.5. Switching performance

\section{Conclusion}

The proposed all-optical switch shows excellent performance for having short switching time and large extinction ratio and relatively large return loss, which are 10.43 ps and $36.8 \mathrm{~dB}, 16.4 \mathrm{~dB}$, respectively. It will be greatly useful for future all-optical computing and signal processing systems. 


\section{Acknowledgements}

This work is supported by the NSFC (Grant No.: 61275043, 60877034, 61307048, 61107049, 61171006), the Guangdong Province NSF (Key project, Grant No.: 82518060-01000004), the Shenzhen Science Bureau (Grant No. 200805, CXB201105050064A, JCYJ20120613115000529).

\section{Reference}

[1] J.J. Bao, J. Xiao, L. Fan, X.X. Li, Y.Y. Hai, T. Zhang and C.B. Yang: Opt. Commun Vol. 329 (2014), p. 109-112

[2] H.J. Ren, J.H. Zhang, Y.L. Qin, K. Liu, J.F. Wu, W.S. Hu, C. Jiang and Y.H. Jin: Opt. Commun Vol. 284 (2011), p. 4073-4077

[3] J.B. Khurgin: Appl. Phys. Lett Vol. 104 (2014), p. 161116

[4] G.Z. Wang, S.F. Zhang, F.A. Umran, X. Cheng, N.N. Dong, C. Darragh, Y. Cheng, L. Zhang, W.J. Blau and J. Wang: Appl. Phys. Lett Vol. 104 (2014), p. 141909

[5] F. Xu, S. Das, Y. Gong, Q. Liu, H.C. Chien, H.Y. Chiu, J. Wu and H. R: Appl. Phys. Lett Vol. 106 (2015), p. 031109

[6] H. Zhang, S. Virally, Q.L. Bao, N. Loh. Godbout, P. K and K. P: Opt. Lett Vol. 37 (2012), p. $1856-1858$

[7] A. Reza, Z.B. Ouyang, Q.Q. Yu, S.C. Ruan: Opt. Express Vol. 22 (2014), p. 14840-14849 